\title{
Drought mitigation in cocoa (Theobroma cacao L.) through developing tolerant hybrids
}

\author{
Baby Juby ${ }^{1}$, Janaki Seifudeen Minimol ${ }^{2 *}$, Basura Suma ${ }^{2}$, Adiyodi Venugopal Santhoshkumar ${ }^{3}$, Joseph Jiji ${ }^{1}$ and \\ Pottekkat Sidharthan Panchami
}

\begin{abstract}
Background: Cocoa, being a shade loving crop cannot withstand long periods of water stress. Breeding for drought tolerance is the need of the hour due to change in climatic condition and extension of crop to non-traditional areas. Hybrids were produced by crossing four tolerant genotypes in all possible combination. The cross GV1 55 x M 13.12 didn't yield any fruit due to cross incompatibility between these genotypes. Various biochemical parameters act as the true indicators to select tolerant and susceptible types. The major biochemical parameters considered after imposing stress included proline, nitrate reductase activity, superoxide dismutase content and glycine betaine.
\end{abstract}

Results: The drought tolerant hybrids were having high amount of proline, superoxide dismutase enzyme and glycine betaine content. Normally, plants having drought stress show low amount of nitrate reductase activity. However, in case of hybrids, the drought tolerant hybrids were having higher NR activity than the susceptible hybrids. The highest amount of NR was found in the control plants kept at fully irrigated conditions.

Conclusions: This experiment showed the role of different biochemical enzymes and osmolytes in giving tolerance to plants during drought stress. Logistic regression analysis selected proline and nitrate reductase as the two biochemical markers for identifying efficient drought tolerant genotypes in the future breeding programmes.

Keywords: Nitrate reductase, Proline, Superoxide dismutase, Logistic regression

\section{Background}

Cocoa is a high industrial valued crop that originated in humid tropics of rain forest and produce cocoa beans which is the only source for chocolate. Cocoa plays a significant role in economics of both producing and consuming countries. In the whole world cocoa is cultivated by around five million farmers and contributes to the subsistence of 40-50 million people [1]. The continuous climate change and limitations in availability of water in several regions of the world, creates a serious threat to plantation industry, especially cocoa as the crop is drought sensitive. Even though cocoa is cultivated under rainfed condition in many cocoa growing countries,

\footnotetext{
*Correspondence: ccrp@kau.in

${ }^{2}$ Cocoa Research Centre, Kerala Agricultural University, Thrissur, India

Full list of author information is available at the end of the article
}

inconsistency in rainfall pattern forms the major constrain. It is estimated that a minimum of 241 of water/ 4-5 days interval is required for the plant to express its maximum potential [2]. The main reason behind this is that it has a very shallow tap root system which enables it to absorb water from surface layers only. The growth and yield of cocoa is influenced by a number of environmental factors, particularly rainfall, temperature and water stress [3].

Drought is defined as a decrease in water inputs or precipitation in an agro/ecosystem over time that is sufficient to result in soil water deficit [4]. Drought stress is the most common environmental stress causing threat to successful production of crop plants. There is a growing concern about the global increase 
in temperature and simultaneous increase in potential evapo- transpiration [5]. Plant water demand may result in increased drought stress during the day and a further deterioration of climatic condition for cocoa. Drought stress normally decreases plant growth by stressing various physiological and biochemical processes in plant, so these parameters can be used as an indicator of stress responses in plants. The plant response to drought stress are usually studied based on physiological parameters, but in recent studies biochemical and antioxidant responses also been proposed as appropriate indicators of drought stress in plants [6,7].

Moreover, due to the increased rate of consumption of cocoa in chocolate industry, the cultivation is extending to non-traditional area. In such area acute water shortage is the most important problem to be addressed. Hence to cope up with the changing climatic scenario and cropping pattern it is essential to breed genotypes that can tolerate water stress. The screening of genotypes based on biochemical parameters helps in developing drought tolerant hybrids which can be used in further breeding programmes. The objective of the present study is to screen and identify cocoa genotypes which can tolerate drought and the role of biochemical parameters in drought stress so as to develop cocoa varieties suitable for drought prone areas.

\section{Materials and methods \\ Experimental site}

The hybridization work was conducted at Cocoa Research Centre (CRC), Kerala Agricultural University, India, using four genotypes identified as tolerant to drought in a preliminary study [8] as parents (Table 1). They were crossed in diallel out of which, the cross GV1 55 x M 13.12 yielded no fruits due to cross incompatibility. The pods matured approximately within 5-6 months; mature pods from each cross were harvested separately and raised in the nursery. Since parents are in heterozygous condition F1 population itself was segregating. Hence each individual was considered as separate hybrids.

Table 1 List of parents used for hybridisation

\begin{tabular}{lll}
\hline SI No & Accession No. & Source \\
\hline 1 & M13.12 & Progeny of pods from Vittal \\
2 & G1 5.9 & T76/1224/1201 (Amazon) \\
3 & G II 19.5 & Progeny of pods from Nileshwar \\
4 & G VI 55 & $\begin{array}{l}\text { Progeny of pods from Cadbury } \\
\text { farm, Chundale }\end{array}$ \\
\hline
\end{tabular}

\section{Drought stress imposition and management}

This study was carried out during 2019-20 in a research greenhouse at Cocoa Research Centre, Vellanikkara located in Thrissur $\left(10^{\circ} 32^{\prime} \mathrm{N}, 76^{\circ} 17^{\prime} \mathrm{E}\right)$. Based on initial vigour, hybrids were selected and subjected to moisture stress following gravimetric method [9]. Seedlings of 5 month old with an average of 15 leaves were selected for drought screening in green house. Each progeny is replicated three times and used in the experiment. Initial trials in CRC indicated that cocoa cannot tolerate water stress less than $40 \%$ field capacity [10]. Hence, $40 \%$ field capacity was maintained for 2 weeks.

A control was also kept at fully irrigated condition representing each of the crosses. Based on the percentage of leaves retained after the drought imposition for 2 weeks, morphological classification of these hybrids was done based on the score chart (Table 2). The humidity and the temperature of the mist chamber were recorded using Berlin's psychrometer on daily basis. Percent of leaves retained was calculated using the formula.

Percentage of leaves retained $=\frac{\text { Number of leaves retained }}{\text { Total number of leaves }} \times 100$

\section{Analysis of biochemical parameters}

Biochemical analysis was carried out using standard procedures after 2 weeks of stress imposition. The parameters considered were proline $(\mu \mathrm{g} / \mathrm{g})[11]$, Nitrate reductase activity (mmol nitrate/g/hr) [12], superoxide dismutase (units/mg protein/g) [13] and glycine betaine $(\mu \mathrm{mol} / \mathrm{g})[14]$. Analysis of variance was done for biochemical analysis for all selected hybrids following completely randomised design (CRD).

\section{Corelation and path coefficient analysis}

The correlation coefficients were calculated to determine the degree of association of characters with percentage of leaves retained. The genotypic coefficients of correlation between character pairs were determined by using the variance and covariance components [15]. The direct and indirect effects of various biochemical parameters was estimated by path coefficient analysis by using the simple correlation coefficient $[16,17]$ and the direct and indirect

Table 2 Score chart depicting the leaves retained in the hybrids

\begin{tabular}{lll}
\hline SI No. & $\begin{array}{l}\text { Percentage of leaves } \\
\text { retained }\end{array}$ & Classification \\
\hline 1 & $0-10$ & Highly Susceptible (HS) \\
2 & $10.1-40$ & Susceptible (S) \\
3 & $40.1-70$ & Tolerant (T) \\
4 & More than 70 & Highly tolerant (HT) \\
\hline
\end{tabular}


effects were grouped into very high $(>1.00)$, high $(0.30$ $0.99)$, medium $(0.20-0.29)$, low $(0.10-0.19)$ and negligible $(0.09-0.00)$ [18].

\section{Binary logistic regression analysis}

Binary logistic regression was carried out to find the relationship between the dependent biochemical parameters over the independent variable, the number of leaves retained. Improvement in selection over the base population was found by the equation,

$$
\text { Per cent improvement over base population }=\frac{\operatorname{Exp}(B)}{1+\operatorname{Exp}(B)} \times 100
$$

\section{Results}

\section{Selection of hybrids and classification}

A total of 1505 hybrid seedlings were raised in the nursery. Based on initial vigour 120 hybrids were selected representing 11 crosses. After imposition of water stress for 2 weeks the percentage of leaves withered was calculated and hybrids were classified as highly tolerant (HT), tolerant $(\mathrm{T})$, susceptible (S) and highly susceptible (HS) as per the score chart given. Table 2. Different biochemical characters recorded from 120 hybrids are statistically analysed and depicted in Table 3.

\section{Effect of water stress on proline content}

In all the 11 crosses, all the tolerant and highly tolerant hybrids indicated high proline content as compared to the susceptible hybrids. The control recorded minimum amount of proline under full irrigated conditions (Table 3).

In the cross M $13.12 \times$ G I 5.9, the highest content was found in hybrid H27 $(1105.64 \mu \mathrm{g} / \mathrm{g})$ and the lowest value was found in H18 $(167.18 \mu \mathrm{g} / \mathrm{g})$. The cross M $13.12 \times \mathrm{G}$ II 19.5 had ten hybrids out of which H38 showed the maximum content of proline of about $2710.82 \mu \mathrm{g} / \mathrm{g}$. Lowest value was found in $\mathrm{H} 40(224.46 \mu \mathrm{g} / \mathrm{g})$. In cross $\mathrm{M}$ $13.12 \times$ G VI 55, the highest content was found in $\mathrm{H} 43$ $(2817.39 \mu \mathrm{g} / \mathrm{g})$ which was a tolerant hybrid and lowest in $\mathrm{H} 44$ of only about $85.52 \mu \mathrm{g} / \mathrm{g}$ of proline indicating its vulnerability to drought stress. The cross G I 5.9 x M 13.12 had six hybrids where H52 had highest proline content of $536.70 \mu \mathrm{g} / \mathrm{g}$ and $\mathrm{H} 54(181.17 \mu \mathrm{g} / \mathrm{g})$ was having lower proline content. In the cross G I 5.9 x G II 19.5, out of 13 hybrids, H63 had the highest proline content of $706.01 \mu \mathrm{g} / \mathrm{g}$ and the lowest was found in H66 $(177.26 \mu \mathrm{g} / \mathrm{g})$. The cross G I 5.9 x G VI $55 \mathrm{had}$ eight hybrids, and the highest content was found in H71 $(1749.05 \mu \mathrm{g} / \mathrm{g})$ while the susceptible hybrid H77 $(169.54 \mu \mathrm{g} / \mathrm{g})$ had the lowest amount. In cross G II 19.5 x M 13.12, the highly tolerant hybrid H85 $(2293.88 \mu \mathrm{g} / \mathrm{g})$ was having the highest amount while the lowest values were found in H91 $(139.87 \mu \mathrm{g} / \mathrm{g})$ which was a susceptible hybrid. In the cross G II 19.5 x G I 5.9, high values for proline was observed in $\mathrm{H} 101(2726.81 \mu \mathrm{g} / \mathrm{g})$ and the lowest in $\mathrm{H} 98(169.18 \mu \mathrm{g} / \mathrm{g})$ which was classified as susceptible. In the cross G II 19.5 x G VI 55), only two hybrids were there, one of which was highly tolerant and the other one was susceptible. The $\mathrm{H} 107$ was having the proline content of $1984.83 \mu \mathrm{g} / \mathrm{g}$ and the susceptible one, H108 was having $339.69 \mu \mathrm{g} / \mathrm{g}$ of proline. The cross G VI 55 x G I 5.9 was having six hybrids and $\mathrm{H} 113$ was having the highest value of proline, $520.85 \mu \mathrm{g} / \mathrm{g}$ and lowest value was found in H114 $(128.55 \mu \mathrm{g} / \mathrm{g})$. In the cross G VI 55 x G II 19.5, highest value was observed in $\mathrm{H} 118(1354.74 \mu \mathrm{g} / \mathrm{g})$ while the lowest value was observed in H116 which was susceptible to drought having only $182.50 \mu \mathrm{g} / \mathrm{g}$ of proline.

When the progenies of all crosses were compared, it was seen that the content ranged from $85.52 \mu \mathrm{g} / \mathrm{g}$ in $\mathrm{H} 44$ (M 13.12 x G VI 55) to $2817.39 \mu \mathrm{g} / \mathrm{g}$ in H43 (M $13.12 \times \mathrm{G}$ VI 55).

\section{Effect of water stress on nitrate reductase activity (NRA)}

Tolerant hybrids had high resistance to drought stress and were able to regulate the nitrate reduction activity even with less water. In this experiment, the cross G VI 55 x G II 19.5 recorded the maximum amount of enzyme activity of about $17.06 \mathrm{mmol}$ nitrate/g/hr. in $\mathrm{H} 115$ which is a tolerant hybrid. However, the susceptible hybrids had very low content of NRA, the lowest being in H17 $(0.51 \mathrm{mmol}$ nitrate $/ \mathrm{g} / \mathrm{hr})$ from the cross M13.12 x G I 5.9. The control plant on the other hand, had the highest value of NRA which was kept under $100 \%$ field capacity.

In the cross between M 13.12 x G I 5.9, 32 hybrids were obtained out of which the H11 showed the maximum nitrate reductase activity of about $12.59 \mathrm{mmol}$ nitrate/g/ $\mathrm{hr}$. while the lowest value of nitrate reductase activity was observed in the susceptible hybrid H17.

The cross M 13.12 x G II 19.5 had hybrids having values as high as $11.74 \mathrm{mmol}$ nitrate/g/hr. in H37 to as low as $2.07 \mathrm{mmol}$ nitrate/g/hr. in H36.In cross M $13.12 \times$ G VI 55, H48 was having the highest NRA content of about $10.89 \mathrm{mmol}$ nitrate $/ \mathrm{g} / \mathrm{hr}$. The lowest value was found in $\mathrm{H} 50$ with only $1.88 \mathrm{mmol}$ nitrate/g/ hr. In the cross between G I 5.9 x M 13.12, highest NRA value was found in $\mathrm{H} 52(8.45 \mathrm{mmol}$ nitrate/g/ hr) which was a tolerant hybrid while the lowest value was found in H54 (1.81 mmol nitrate/g/hr). The cross G I $5.9 \times$ G II 19.5 had H64 with the highest NRA value of $9.48 \mathrm{mmol}$ nitrate $/ \mathrm{g} / \mathrm{hr}$. while the lowest value for NRA was $1.41 \mathrm{mmol}$ nitrate $/ \mathrm{g} / \mathrm{hr}$. found in H59.In the cross between G I $5.9 \times$ G VI 55, the highest NR activity was found in $\mathrm{H} 76$ with $10.41 \mathrm{mmol}$ nitrate/g/hr. which is a highly tolerant hybrid. The lowest NR activity was found in $\mathrm{H} 73$ having $3.65 \mathrm{mmol}$ nitrate/g/hr. which 
Table 3 Biochemical parameters of cocoa hybrid seedlings

\begin{tabular}{|c|c|c|c|c|c|c|}
\hline SI. No. & Hybrids & $\begin{array}{l}\text { Reaction to } \\
\text { drought }\end{array}$ & Proline $(\mu \mathrm{g} / \mathrm{g})$ & $\begin{array}{l}\text { NRA } \\
\text { (mmol nitrate/g/ } \\
\text { hr) }\end{array}$ & $\begin{array}{l}\text { SOD } \\
\text { (units/mg } \\
\text { protein/g) }\end{array}$ & $\begin{array}{l}\text { Glycine } \\
\text { betaine } \\
(\mu \mathrm{mol} / \mathrm{g})\end{array}$ \\
\hline \multicolumn{7}{|c|}{ (I). M13.12 X G I 5.9} \\
\hline 1 & $\mathrm{H} 1$ & $S$ & 269.08 & 1.34 & 0.208 & 6.30 \\
\hline 2 & $\mathrm{H} 2$ & $\mathrm{~T}$ & 695.36 & 5.60 & 0.373 & 7.48 \\
\hline 3 & $\mathrm{H} 3$ & S & 181.96 & 4.16 & 0.186 & 4.42 \\
\hline 4 & $\mathrm{H} 4$ & $S$ & 219.44 & 2.28 & 0.205 & 6.03 \\
\hline 5 & $\mathrm{H} 5$ & $S$ & 247.51 & 2.14 & 0.209 & 5.28 \\
\hline 6 & $\mathrm{H} 6$ & $\mathrm{~S}$ & 298.52 & 3.82 & 0.210 & 6.22 \\
\hline 7 & $\mathrm{H} 7$ & $\mathrm{~S}$ & 304.19 & 3.24 & 0.207 & 6.21 \\
\hline 8 & $\mathrm{H} 8$ & $S$ & 354.34 & 4.04 & 0.188 & 6.15 \\
\hline 9 & $\mathrm{H} 9$ & $\mathrm{~T}$ & 996.41 & 8.59 & 0.327 & 6.35 \\
\hline 10 & $\mathrm{H} 10$ & $\mathrm{~T}$ & 440.93 & 11.92 & 0.381 & 6.77 \\
\hline 11 & $\mathrm{H} 11$ & $\mathrm{~T}$ & 452.91 & 12.59 & 0.317 & 7.03 \\
\hline 12 & $\mathrm{H} 12$ & $\mathrm{~T}$ & 539.50 & 6.94 & 0.351 & 7.09 \\
\hline 13 & $\mathrm{H} 13$ & $\mathrm{~T}$ & 446.25 & 6.25 & 0.318 & 7.43 \\
\hline 14 & $\mathrm{H} 14$ & $S$ & 293.06 & 4.03 & 0.210 & 5.33 \\
\hline 15 & $\mathrm{H} 15$ & S & 254.43 & 2.43 & 0.159 & 6.27 \\
\hline 16 & $\mathrm{H} 16$ & $S$ & 310.02 & 2.19 & 0.128 & 5.25 \\
\hline 17 & $\mathrm{H} 17$ & $S$ & 174.24 & 0.51 & 0.210 & 5.39 \\
\hline 18 & $\mathrm{H} 18$ & $S$ & 167.18 & 2.56 & 0.209 & 6.04 \\
\hline 19 & $\mathrm{H} 19$ & $\mathrm{~S}$ & 175.86 & 3.48 & 0.211 & 6.26 \\
\hline 20 & $\mathrm{H} 2 \mathrm{O}$ & $T$ & 418.28 & 7.12 & 0.318 & 6.78 \\
\hline 21 & $\mathrm{H} 21$ & $S$ & 290.00 & 4.22 & 0.164 & 5.71 \\
\hline 22 & $\mathrm{H} 22$ & $S$ & 189.29 & 3.35 & 0.212 & 5.94 \\
\hline 23 & $\mathrm{H} 23$ & $S$ & 173.17 & 4.03 & 0.147 & 5.32 \\
\hline 24 & $\mathrm{H} 24$ & $\mathrm{~T}$ & 468.86 & 5.85 & 0.351 & 7.99 \\
\hline 25 & $\mathrm{H} 25$ & $\mathrm{~T}$ & 438.26 & 8.39 & 0.325 & 7.43 \\
\hline 26 & $\mathrm{H} 26$ & $T$ & 504.07 & 5.08 & 0.321 & 11.64 \\
\hline 27 & $\mathrm{H} 27$ & HT & 1105.64 & 10.93 & 0.364 & 10.43 \\
\hline 28 & $\mathrm{H} 28$ & $\mathrm{~T}$ & 479.56 & 7.13 & 0.343 & 7.81 \\
\hline 29 & $\mathrm{H} 29$ & $\mathrm{~T}$ & 479.54 & 5.16 & 0.319 & 6.43 \\
\hline 30 & $\mathrm{H} 30$ & $S$ & 340.35 & 1.02 & 0.191 & 5.36 \\
\hline 31 & $\mathrm{H} 31$ & $\mathrm{~T}$ & 490.21 & 5.11 & 0.345 & 8.77 \\
\hline \multirow[t]{4}{*}{32} & $\mathrm{H} 32$ & $S$ & 209.81 & 2.65 & 0.164 & 5.32 \\
\hline & Control & & 61.33 & 17.03 & 0.023 & 3.22 \\
\hline & CV (\%) & & 4.83 & 14.60 & 16.93 & 10.36 \\
\hline & $C D$ & $0.05 \%$ & 30.54 & 1.12 & 0.07 & 1.12 \\
\hline \multicolumn{7}{|c|}{ (II). M13.12 X G II 19.5} \\
\hline 33 & $\mathrm{H} 33$ & $\mathrm{~T}$ & 671.38 & 6.36 & 0.316 & 9.46 \\
\hline 34 & $\mathrm{H} 34$ & $\mathrm{~T}$ & 547.57 & 6.79 & 0.315 & 9.68 \\
\hline 35 & $\mathrm{H} 35$ & S & 359.00 & 2.70 & 0.195 & 6.73 \\
\hline 36 & $\mathrm{H} 36$ & $\mathrm{~S}$ & 459.65 & 2.07 & 0.203 & 6.06 \\
\hline 37 & $\mathrm{H} 37$ & $\mathrm{~T}$ & 523.52 & 11.74 & 0.319 & 8.79 \\
\hline 38 & $\mathrm{H} 38$ & HT & 2710.82 & 7.16 & 0.332 & 9.52 \\
\hline 39 & $\mathrm{H} 39$ & $\mathrm{~T}$ & 522.28 & 6.59 & 0.322 & 8.66 \\
\hline 40 & $\mathrm{H} 40$ & S & 224.46 & 3.62 & 0.197 & 7.34 \\
\hline 41 & $\mathrm{H} 41$ & $\mathrm{~S}$ & 265.09 & 4.30 & 0.184 & 6.02 \\
\hline \multirow[t]{2}{*}{42} & $\mathrm{H} 42$ & $\mathrm{~T}$ & 619.43 & 8.52 & 0.311 & 9.35 \\
\hline & Control & & 75.73 & 19.98 & 0.064 & 2.84 \\
\hline
\end{tabular}


Table 3 (continued)

\begin{tabular}{|c|c|c|c|c|c|c|}
\hline SI. No. & Hybrids & $\begin{array}{l}\text { Reaction to } \\
\text { drought }\end{array}$ & Proline $(\mu \mathrm{g} / \mathrm{g})$ & $\begin{array}{l}\text { NRA } \\
\text { (mmol nitrate/g/ } \\
\text { hr) }\end{array}$ & $\begin{array}{l}\text { SOD } \\
\text { (units/mg } \\
\text { protein/g) }\end{array}$ & $\begin{array}{l}\text { Glycine } \\
\text { betaine } \\
\text { ( } \mu \mathrm{mol} / \mathrm{g})\end{array}$ \\
\hline & CV (\%) & & 2.59 & 15.25 & 13.65 & 9.34 \\
\hline & $C D$ & $0.05 \%$ & 34.72 & 1.55 & 0.06 & 1.30 \\
\hline \multicolumn{7}{|c|}{ (III). M13.12 X G VI 55} \\
\hline 43 & $\mathrm{H} 43$ & $\mathrm{HT}$ & 2817.39 & 9.75 & 0.337 & 8.90 \\
\hline 44 & $\mathrm{H} 44$ & $\mathrm{HS}$ & 85.52 & 3.39 & 0.145 & 6.70 \\
\hline 45 & $\mathrm{H} 45$ & $S$ & 163.72 & 3.02 & 0.166 & 5.29 \\
\hline 46 & $\mathrm{H} 46$ & $\mathrm{~T}$ & 643.40 & 6.40 & 0.362 & 9.66 \\
\hline 47 & $\mathrm{H} 47$ & S & 111.90 & 3.49 & 0.215 & 6.98 \\
\hline 48 & $\mathrm{H} 48$ & $\mathrm{~T}$ & 494.21 & 10.89 & 0.334 & 9.87 \\
\hline 49 & $\mathrm{H} 49$ & S & 400.96 & 4.12 & 0.193 & 6.87 \\
\hline 50 & $\mathrm{H} 50$ & S & 247.77 & 1.88 & 0.215 & 7.06 \\
\hline \multirow[t]{4}{*}{51} & $\mathrm{H} 51$ & S & 170.51 & 4.05 & 0.215 & 7.13 \\
\hline & Control & & 65.60 & 17.27 & 0.035 & 2.82 \\
\hline & CV (\%) & & 2.65 & 13.85 & 18.88 & 11.74 \\
\hline & $C D$ & $0.05 \%$ & 25.98 & 1.24 & 0.08 & 1.53 \\
\hline \multicolumn{7}{|c|}{ (IV). G I 5.9 x M 13.12} \\
\hline 52 & $\mathrm{H} 52$ & $\mathrm{~T}$ & 536.70 & 8.45 & 0.259 & 8.10 \\
\hline 53 & $\mathrm{H} 53$ & S & 292.40 & 2.96 & 0.167 & 6.43 \\
\hline 54 & $\mathrm{H} 54$ & S & 181.17 & 1.81 & 0.172 & 5.88 \\
\hline 55 & $\mathrm{H} 55$ & $S$ & 386.31 & 3.92 & 0.156 & 5.36 \\
\hline 56 & H56 & S & 235.78 & 3.88 & 0.173 & 6.57 \\
\hline \multirow[t]{4}{*}{57} & $\mathrm{H} 57$ & $\mathrm{~T}$ & 498.21 & 4.25 & 0.222 & 7.97 \\
\hline & Control & & 85.70 & 12.95 & 0.027 & 3.16 \\
\hline & CV (\%) & & 6.01 & 15.02 & 18.31 & 10.21 \\
\hline & $C D$ & $0.05 \%$ & 38.00 & 1.13 & 0.06 & 1.22 \\
\hline \multicolumn{7}{|c|}{ (V). G I 5.9 X G II 19.5} \\
\hline 58 & $\mathrm{H} 58$ & $S$ & 218.46 & 3.91 & 0.218 & 6.83 \\
\hline 59 & H59 & $S$ & 333.96 & 1.41 & 0.217 & 7.18 \\
\hline 60 & $\mathrm{H} 60$ & S & 326.36 & 3.62 & 0.162 & 7.34 \\
\hline 61 & $\mathrm{H} 61$ & S & 311.71 & 2.09 & 0.166 & 7.32 \\
\hline 62 & $\mathrm{H} 62$ & $\mathrm{~T}$ & 568.81 & 8.39 & 0.248 & 8.93 \\
\hline 63 & $\mathrm{H} 63$ & $\mathrm{~T}$ & 706.01 & 8.66 & 0.241 & 9.52 \\
\hline 64 & $\mathrm{H} 64$ & $\mathrm{~T}$ & 679.37 & 9.48 & 0.243 & 8.61 \\
\hline 65 & $\mathrm{H} 65$ & S & 325.70 & 4.00 & 0.213 & 7.11 \\
\hline 66 & H66 & S & 177.26 & 1.42 & 0.222 & 7.17 \\
\hline 67 & $\mathrm{H} 67$ & $\mathrm{~T}$ & 699.35 & 9.05 & 0.237 & 8.32 \\
\hline 68 & H68 & $\mathrm{T}$ & 684.66 & 8.56 & 0.276 & 9.38 \\
\hline 69 & $\mathrm{H} 69$ & S & 196.48 & 4.03 & 0.173 & 7.28 \\
\hline \multirow[t]{4}{*}{70} & $\mathrm{H} 70$ & S & 167.84 & 3.32 & 0.204 & 6.45 \\
\hline & Control & & 138.00 & 15.78 & 0.050 & 3.34 \\
\hline & CV (\%) & & 6.84 & 8.68 & 18.23 & 9.82 \\
\hline & $C D(0.05)$ & & 47.62 & 0.76 & 0.07 & 1.29 \\
\hline \multicolumn{7}{|c|}{ (VI). G I 5.9 X G VI 55} \\
\hline 71 & $\mathrm{H} 71$ & $\mathrm{HT}$ & 1749.05 & 9.94 & 0.256 & 9.56 \\
\hline 72 & $\mathrm{H} 72$ & S & 319.70 & 4.53 & 0.227 & 7.05 \\
\hline 73 & $\mathrm{H} 73$ & S & 395.63 & 3.65 & 0.234 & 7.07 \\
\hline 74 & $\mathrm{H} 74$ & HT & 1555.89 & 9.83 & 0.254 & 8.85 \\
\hline 75 & $\mathrm{H} 75$ & $\mathrm{~T}$ & 450.25 & 10.26 & 0.272 & 9.38 \\
\hline
\end{tabular}


Table 3 (continued)

\begin{tabular}{|c|c|c|c|c|c|c|}
\hline SI. No. & Hybrids & $\begin{array}{l}\text { Reaction to } \\
\text { drought }\end{array}$ & Proline $(\mu \mathrm{g} / \mathrm{g})$ & $\begin{array}{l}\text { NRA } \\
\text { (mmol nitrate/g/ } \\
\text { hr) }\end{array}$ & $\begin{array}{l}\text { SOD } \\
\text { (units/mg } \\
\text { protein/g) }\end{array}$ & $\begin{array}{l}\text { Glycine } \\
\text { betaine } \\
(\mu \mathrm{mol} / \mathrm{g})\end{array}$ \\
\hline 76 & $\mathrm{H} 76$ & HT & 1126.96 & 10.41 & 0.266 & 8.63 \\
\hline 77 & $\mathrm{H} 77$ & S & 169.54 & 4.50 & 0.191 & 6.34 \\
\hline \multirow[t]{4}{*}{78} & $\mathrm{H} 78$ & S & 224.99 & 4.28 & 0.218 & 6.30 \\
\hline & Control & & 101.33 & 16.61 & 0.066 & 3.18 \\
\hline & CV (\%) & & 4.54 & 6.58 & 12.65 & 9.90 \\
\hline & $C D$ & $0.05 \%$ & 35.13 & 0.82 & NS & 1.35 \\
\hline \multicolumn{7}{|c|}{ (VII). G II 19.5 x M 13.12} \\
\hline 79 & $\mathrm{H} 79$ & $\mathrm{~T}$ & 853.88 & 7.18 & 0.225 & 8.87 \\
\hline 80 & $\mathrm{H} 80$ & S & 325.03 & 4.62 & 0.177 & 6.78 \\
\hline 81 & $\mathrm{H} 81$ & $\mathrm{~T}$ & 498.21 & 14.27 & 0.209 & 8.91 \\
\hline 82 & H82 & $\mathrm{T}$ & 692.69 & 8.54 & 0.197 & 8.70 \\
\hline 83 & H83 & $\mathrm{T}$ & 454.91 & 7.48 & 0.224 & 8.66 \\
\hline 84 & $\mathrm{H} 84$ & $S$ & 269.08 & 5.27 & 0.181 & 7.07 \\
\hline 85 & H85 & $\mathrm{HT}$ & 2293.88 & 7.72 & 0.227 & 8.43 \\
\hline 86 & H86 & $S$ & 374.99 & 5.20 & 0.182 & 7.06 \\
\hline 87 & $\mathrm{H} 87$ & S & 363.66 & 4.24 & 0.170 & 7.51 \\
\hline 88 & $\mathrm{H} 88$ & $\mathrm{~T}$ & 625.80 & 11.82 & 0.199 & 10.08 \\
\hline 89 & H89 & $S$ & 295.73 & 3.98 & 0.183 & 7.31 \\
\hline 90 & H9O & $\mathrm{HT}$ & 1689.10 & 10.63 & 0.205 & 8.89 \\
\hline 91 & H91 & $S$ & 139.87 & 2.80 & 0.184 & 7.55 \\
\hline 92 & H92 & S & 303.72 & 5.09 & 0.180 & 7.20 \\
\hline 93 & H93 & S & 338.75 & 4.03 & 0.133 & 6.93 \\
\hline 94 & $\mathrm{H} 94$ & $\mathrm{~T}$ & 447.59 & 6.90 & 0.217 & 9.17 \\
\hline 95 & H95 & S & 208.47 & 4.02 & 0.162 & 6.84 \\
\hline \multirow[t]{4}{*}{96} & H96 & $\mathrm{HT}$ & 2011.47 & 7.80 & 0.215 & 9.55 \\
\hline & Control & & 95.47 & 15.49 & 0.042 & 3.51 \\
\hline & CV (\%) & & 4.52 & 7.95 & 19.43 & 10.15 \\
\hline & $C D$ & $0.05 \%$ & 43.84 & 0.89 & NS & 1.36 \\
\hline \multicolumn{7}{|c|}{ (VIII). G II 19.5 x G I 5.9} \\
\hline 97 & $\mathrm{H} 97$ & $\mathrm{HT}$ & 1490.62 & 15.45 & 0.265 & 9.46 \\
\hline 98 & H98 & S & 169.18 & 4.76 & 0.209 & 7.71 \\
\hline 99 & H99 & S & 363.66 & 4.80 & 0.213 & 7.20 \\
\hline 100 & $\mathrm{H} 100$ & S & 215.67 & 5.13 & 0.199 & 7.90 \\
\hline 101 & H101 & $\mathrm{HT}$ & 2726.81 & 9.80 & 0.270 & 9.70 \\
\hline 102 & $\mathrm{H} 102$ & $\mathrm{~T}$ & 454.25 & 9.90 & 0.248 & 11.31 \\
\hline 103 & $\mathrm{H} 103$ & $\mathrm{~T}$ & 500.87 & 8.09 & 0.247 & 13.79 \\
\hline 104 & $\mathrm{H} 104$ & $\mathrm{~T}$ & 543.50 & 8.86 & 0.239 & 9.80 \\
\hline 105 & $\mathrm{H} 105$ & S & 171.93 & 5.92 & 0.207 & 8.02 \\
\hline \multirow[t]{4}{*}{106} & $\mathrm{H} 106$ & S & 162.52 & 5.49 & 0.154 & 7.57 \\
\hline & Control & & 90.13 & 18.81 & 0.017 & 3.33 \\
\hline & CV (\%) & & 4.70 & 8.73 & 18.63 & 9.74 \\
\hline & $C D$ & $0.05 \%$ & 54.47 & 1.16 & NS & 1.53 \\
\hline \multicolumn{7}{|c|}{ (IX). G II 19.5 x G VI 55} \\
\hline 107 & $\mathrm{H} 107$ & $\mathrm{HT}$ & 1984.83 & 12.98 & 0.163 & 9.34 \\
\hline \multirow[t]{4}{*}{108} & $\mathrm{H} 108$ & S & 339.69 & 6.65 & 0.106 & 7.18 \\
\hline & Control & & 69.33 & 16.66 & 0.065 & 2.43 \\
\hline & CV (\%) & & 2.75 & 4.97 & 18.99 & 5.58 \\
\hline & $C D$ & $0.05 \%$ & 72.38 & 1.11 & NS & 1.04 \\
\hline
\end{tabular}


Table 3 (continued)

\begin{tabular}{|c|c|c|c|c|c|c|}
\hline SI. No. & Hybrids & $\begin{array}{l}\text { Reaction to } \\
\text { drought }\end{array}$ & Proline $(\mu \mathrm{g} / \mathrm{g})$ & $\begin{array}{l}\text { NRA } \\
\text { (mmol nitrate/g/ } \\
\text { hr) }\end{array}$ & $\begin{array}{l}\text { SOD } \\
\text { (units/mg } \\
\text { protein/g) }\end{array}$ & $\begin{array}{l}\text { Glycine } \\
\text { betaine } \\
\text { ( } \mu \mathrm{mol} / \mathrm{g})\end{array}$ \\
\hline \multicolumn{7}{|c|}{ (X). G VI 55 x G I 5.9} \\
\hline 109 & $\mathrm{H} 109$ & S & 308.91 & 4.12 & 0.185 & 6.93 \\
\hline 110 & $\mathrm{H} 110$ & $S$ & 151.99 & 3.57 & 0.136 & 7.66 \\
\hline 111 & $\mathrm{H} 111$ & $\mathrm{~T}$ & 424.27 & 6.68 & 0.207 & 8.94 \\
\hline 112 & $\mathrm{H} 112$ & $\mathrm{~T}$ & 411.35 & 6.38 & 0.221 & 10.61 \\
\hline 113 & $\mathrm{H} 113$ & $\mathrm{~T}$ & 520.85 & 7.24 & 0.207 & 9.93 \\
\hline \multirow[t]{4}{*}{114} & $\mathrm{H} 114$ & S & 128.55 & 4.63 & 0.149 & 7.42 \\
\hline & Control & & 74.67 & 14.76 & 0.025 & 3.03 \\
\hline & CV (\%) & & 7.23 & 6.15 & 17.34 & 11.23 \\
\hline & $C D$ & $0.05 \%$ & 41.74 & 0.59 & 0.06 & 1.17 \\
\hline \multicolumn{7}{|c|}{ (XI). G VI 55 x G II 19.5} \\
\hline 115 & $\mathrm{H} 115$ & $\mathrm{~T}$ & 520.85 & 17.06 & 0.209 & 6.52 \\
\hline 116 & $\mathrm{H} 116$ & S & 182.50 & 4.10 & 0.140 & 4.42 \\
\hline 117 & $\mathrm{H} 117$ & $\mathrm{~T}$ & 743.18 & 11.35 & 0.202 & 6.43 \\
\hline 118 & $\mathrm{H} 118$ & $\mathrm{HT}$ & 1354.75 & 8.76 & 0.227 & 8.50 \\
\hline 119 & $\mathrm{H} 119$ & $\mathrm{~T}$ & 412.95 & 7.56 & 0.163 & 9.23 \\
\hline \multirow[t]{4}{*}{120} & $\mathrm{H} 120$ & $T$ & 507.53 & 6.98 & 0.173 & 5.67 \\
\hline & Control & & 94.00 & 23.05 & 0.032 & 3.18 \\
\hline & CV (\%) & & 5.21 & 10.22 & 18.49 & 8.92 \\
\hline & CD (0.05\%) & & 57.48 & 1.74 & NS & 1.08 \\
\hline
\end{tabular}

$\mathrm{CD}$ (critical difference is used to compare means of different treatments that have equal number of replications)

is susceptible hybrid. In cross G II 19.5 x M 13.12, H81 showed the maximum NR activity of $14.27 \mathrm{mmol}$ nitrate/g/hr. and the lowest value $(3.98 \mathrm{mmol}$ nitrate $/ \mathrm{g} /$ hr) was observed inthe susceptible hybrid H89.The cross G II 19.5 x G I 5.9 had hybrid having the highest value in NR activity (H97) with a value of $15.45 \mathrm{mmol}$ nitrate $/ \mathrm{g} / \mathrm{hr}$. and the lowest $(4.76 \mathrm{mmol}$ nitrate $/ \mathrm{g} /$ hr) was reported in H98.The cross G II 19.5 x G VI 55 had two hybrids, the high NR value was observed in the tolerant hybrid $\mathrm{H} 107$ (12.98 mmol nitrate/g/ hr) and the low value of NR activity was observed in H108 (6.65 mmol nitrate $/ \mathrm{g} / \mathrm{hr}$ ). In the cross G VI $55 \mathrm{x}$ G I 5.9, the tolerant hybrids were having the high NR activity, the highest being $\mathrm{H} 111$ (6.68 $\mathrm{mmol}$ nitrate $/ \mathrm{g} /$ $\mathrm{hr})$, and lowest in $\mathrm{H} 110 \quad(3.57 \mathrm{mmol}$ nitrate/g/hr). The cross G VI $55 \times$ G II 19.5 had values as high as $17.06 \mathrm{mmol}$ nitrate/g/hr. in $\mathrm{H} 115$ while the lowest value was observed in $\mathrm{H} 116$ having $4.10 \mathrm{mmol}$ nitrate/g/hr. nitrate activity.

\section{Effect of water stress on superoxide dismutase}

In cross M13.12 x G I 5.9, the highest value was observed in $\mathrm{H} 10$ ( 0.381 units $/ \mathrm{mg}$ protein $/ \mathrm{g})$ while the lowest value was observed in $\mathrm{H} 16$ (0.128 units $/ \mathrm{mg}$ protein $/ \mathrm{g})$. In the cross M 13.12 x G II 19.5, highest SOD value was found in H38 (0.332 units/mg protein/g) which is a highly tolerant hybrid while the lowest was H41 (0.184 units/mg protein/g). In the cross M $13.12 \times$ G VI 55, the highest SOD value was found in $\mathrm{H} 46$ ( 0.362 units/mg protein/g). The lowest SOD value was in hybrid H45 (0.166 units/ mg protein/g) which was highly susceptible. The cross G I $5.9 \times$ M 13.12 had the highest SOD values of about 0.259 units $/ \mathrm{mg}$ protein/g expressed in hybrid H52 while the lowest values was found in H55 (0.156 units/mg protein/g). In cross G I 5.9 x G II 19.5, the highest value was present in hybrid $\mathrm{H} 68$ (0.276 units/mg protein/g). The lowest value obtained was in hybrid H60 (0.162 units/ mg protein/g). The cross G I 5.9 x G VI 55 had high SOD activity in hybrid $\mathrm{H} 75$ ( 0.272 units/mg protein $/ \mathrm{g}$ ) whereas lowest SOD value was found in H77 (0.191 units/mg protein/g). In cross G II 19.5 x M 13.12, SOD values was highest in $\mathrm{H} 85$ (0.227 units $/ \mathrm{mg}$ protein $/ \mathrm{g})$. The lowest SOD value was found in $\mathrm{H} 93$ ( $0.133 \mathrm{units} / \mathrm{mg}$ protein $/ \mathrm{g}$ ). In the cross G II 19.5 x G I 5.9, hybrid H101 (0.270 units/ $\mathrm{mg}$ protein $/ \mathrm{g}$ ) had the highest value and the lowest was found in H106 (0.154 units/mg protein/g). In the cross G II $19.5 \times$ G VI 55, two hybrids were obtained in which the hybrid $\mathrm{H} 107$ was highly tolerant hybrid having high SOD content of 0.163 units $/ \mathrm{mg}$ protein/g whereas the susceptible hybrid was having 0.106 units $/ \mathrm{mg}$ protein/g of SOD activity. In the cross G VI 55 x G I 5.9, high SOD value was found in hybridH112 (0.221 units/mg protein/g). The 
low SOD value was found in hybrid H110 (0.136 units/mg protein/g). In cross G VI 55 x G II 19.5, the highest value was found in hybrid H118 (0.227 units/mg protein/g) while the lowest value was found in hybrid H116 ( 0.140 units $/ \mathrm{mg}$ protein/g) which is a susceptible hybrid.

H10 (M13.12 x G I 5.9) recorded the high SOD content of about 0.381 units $/ \mathrm{mg}$ protein/g which was a tolerant hybrid and the lowest content was observed in a susceptible hybrid, H108 (G II 19.5 x G VI 55) of about 0.106 units/mg protein/g.

\section{Effect of water stress on glycine betaine (GB)}

When the hybrids of present study were analysed, the tolerant hybrid, H103 (G II 19.5 x G I 5.9) was having high amount of glycine betaine of about $13.79 \mu \mathrm{mol} / \mathrm{g}$ and the susceptible hybrid, $\mathrm{H} 3(4.42 \mu \mathrm{mol} / \mathrm{g})$ of the cross M13.12 x G I 5.9 recorded the least. All the tolerant hybrids had high glycine betaine content as compared to the susceptible hybrids. However, the control which was kept under fully irrigated condition had least amount of glycine betaine among the crosses indicating the accumulation of glycine betaine under drought stress conditions. In cross M 13.12 x G I 5.9, the highest glycine betaine value was found in $\mathrm{H} 26(11.64 \mu \mathrm{mol} / \mathrm{g})$ and the lowest value was observed in $\mathrm{H} 3(4.42 \mu \mathrm{mol} / \mathrm{g})$. In cross M $13.12 \times$ G II 19.5, the highest value was found in hybrid H34 $(9.68 \mu \mathrm{mol} / \mathrm{g})$ whereas the lowest value was observed in $\mathrm{H} 35(6.73 \mu \mathrm{mol} / \mathrm{g})$. In cross M13.12 x G VI 55 the highest value was observed in $\mathrm{H} 48(9.87 \mu \mathrm{mol} / \mathrm{g})$ and the $\mathrm{H} 45(5.29 \mu \mathrm{mol} / \mathrm{g})$ recorded the lowest glycine betaine value.

In cross G I 5.9 x M 13.12, high GB values were observed in $\mathrm{H} 52(8.10 \mu \mathrm{mol} / \mathrm{g})$ while $\mathrm{H} 55(5.36 \mu \mathrm{mol} / \mathrm{g})$ had the lowest value. In cross G I 5.9 x G II 19.5, the highest value was observed in $\mathrm{H} 63(9.52 \mu \mathrm{mol} / \mathrm{g})$ to as low as $6.45 \mu \mathrm{mol} / \mathrm{g}$ in H70.In cross G I 5.9 x G VI 55, the highest value was observed in hybrid $\mathrm{H} 71(9.56 \mu \mathrm{mol} / \mathrm{g})$. Lowest values were observed in $\mathrm{H} 73(7.07 \mu \mathrm{mol} / \mathrm{g})$. In cross G II 19.5 x M 13.12, highest GB value was observed in H88 $(10.08 \mu \mathrm{mol} / \mathrm{g})$ while the lowest value was observed in $\mathrm{H} 80(6.78 \mu \mathrm{mol} / \mathrm{g})$. In the cross between G II $19.5 \times$ G I 5.9, $\mathrm{H} 103(13.79 \mu \mathrm{mol} / \mathrm{g})$ recorded the highest value while the lowest value was observed in $\mathrm{H} 99(7.20 \mu \mathrm{mol} / \mathrm{g})$. The cross G II 19.5 x G VI 55 had two hybrids, the hybrid $\mathrm{H} 107$ had high GB value of $9.34 \mu \mathrm{mol} / \mathrm{g}$ and the other hybrid, H108 which is the susceptible one, had $7.18 \mu \mathrm{mol} / \mathrm{g}$ of GB. In cross G VI 55 x G I 5.9, H112 was having the highest GB content of about $10.61 \mu \mathrm{mol} / \mathrm{g}$ while the lowest value was observed in $\mathrm{H} 109$ $(6.93 \mu \mathrm{mol} / \mathrm{g})$. In the cross G VI $55 \times$ G II 19.5, the highest value was observed in hybrid $\mathrm{H} 119(9.23 \mu \mathrm{mol} / \mathrm{g})$ while the lowest value was observed in $\mathrm{H} 116(4.42 \mu \mathrm{mol} / \mathrm{g})$.

\section{Corelation and path coefficient analysis}

All the biochemical parameters estimated showed positive corelation with dependant variable (percentage of leaves retained). Among the biochemical characters, proline showed maximum correlation with the dependent variable, percentage of leaves retained (0.777) (Table 4).

High positive effect on the percentage of leaves retained in path analysis result is a direct measure of plant's tolerance to drought. It was expressed by proline $(0.386)$ and low direct effect was shown by nitrate reductase activity (0.166) (Fig. 1). Negligible direct effects were found in superoxide dismutase (0.063), and negative but negligible effects were shown by glycine betaine $(-0.016)$.

\section{Binary logistic regression analysis}

The positive and comparable value of odds ratio Exp (B) and positive correlation indicated that proline content and NRA content had a positive correlation with drought tolerance and also these characters expressed a significant value of less than 0.05 which is the constant indicating the $95 \%$ accuracy with the results.

Based on the Exp (B) value from regression model, expressed percentage for drought tolerance over the base population was calculated and it was found that if selection is based on proline, a new population from the base population will express $51.55 \%$ improvement regarding the tolerance. In case of NRA, a new population will show $87.48 \%$ improvementover the base population (Table 5).

\section{Discussion}

Drought stress is one of the important problems which adversely affects the performance of different crop plants worldwide. Various strategies are being adopted to cop up with adverse conditions. Development of drought tolerant cultivars is prerequisite to encounter

Table 4 Correlation among drought tolerant contributing characters of hybrids

\begin{tabular}{lllll}
\hline & V1 & V2 & V3 & V4 \\
\hline V1 & 1 & & & \\
V2 & $0.534^{* *}$ & 1 & & \\
V3 & $0.353^{* *}$ & $0.440^{* *}$ & 1 & \\
V4 & $0.458^{* *}$ & $0.581^{* *}$ & $0.441^{* *}$ & 1 \\
\hline
\end{tabular}

\footnotetext{
** Correlation significant at 0.01 level

V 1 - Proline $(\mu \mathrm{g} / \mathrm{g})$

V 2 - Nitrate reductase activity ( $\mathrm{mmol}$ nitrate $/ \mathrm{g} / \mathrm{hr}$ )

V 3 - Superoxide dismutase (units/mg protein/g)

V 4 - Glycine betaine $(\mu \mathrm{mol} / \mathrm{g})$
} 


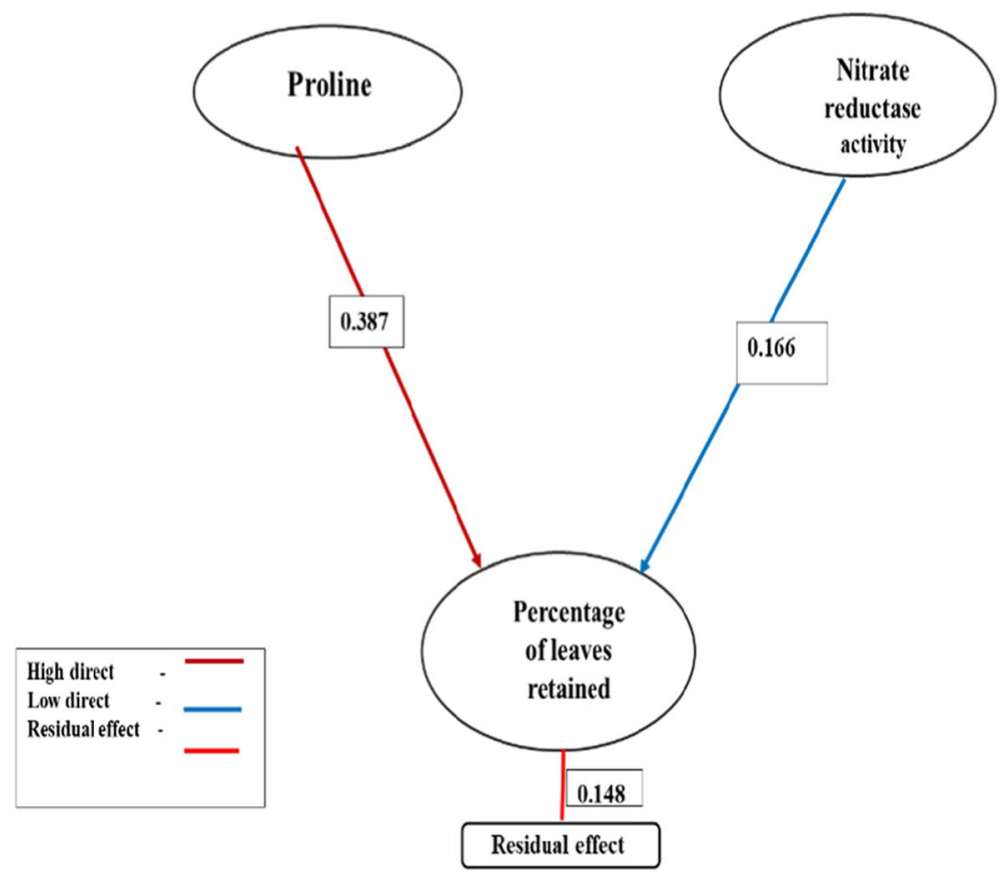

Fig. 1 Path diagram for the biochemical characters having direct effects on percent of leaves retained

Table 5 Logistic estimate of characters influencing drought tolerance in cocoa

\begin{tabular}{lllllll}
\hline Variables & Coefficient & Standard error & Wald & Significance & Exp(B) & $\begin{array}{l}\text { Expected per cent } \\
\text { improvement over } \\
\text { population (\%) }\end{array}$ \\
\hline Proline $^{\mathrm{a}}$ & 0.062 & 0.011 & 31.019 & 0.001 & 1.064 & 51.55 \\
$\begin{array}{l}\text { Nitrate reductase } \\
\text { activity }^{\mathrm{a}}\end{array}$ & 1.944 & 0.233 & 69.914 & 0.001 & 6.990 & 87.48 \\
\hline
\end{tabular}

a Significant value less than 0.05

the existing drought stress on sustainable basis. In the present study selection of hybrids were done based on initial vigor and stress imposition was carried out. In cocoa initial vigour is directly correlated to yield [19].

In general, plants employ various methods to promote tolerance to drought stress. To assess the degree of tolerance in plants, various drought-related indicators are used, such as morphological, physiological and biochemical parameters [20]. In the present study evaluation and screening of genotypes under drought stress were done based on biochemical parameters.

Proline is an important amino acid found in proteins. It has a significant contribution in drought tolerance. Under drought stress, a higher accumulation of proline was recorded in the study, which might be due to osmotic adjustment. Even within a single cross, highly tolerant as well as susceptible hybrids were found. This is because parents are heterozygous in nature [21]. The analysis of proline clearly indicated that resistant plants were high in proline content compared to susceptible ones. A clearcut difference was observed between highly tolerant and susceptible genotypes indicating that proline plays an important role in drought tolerance of cocoa. High levels of proline enabled the plant to maintain low water potentials. Apart from acting as an osmolyte for osmotic adjustment, proline contributes to stabilizing sub-cellular structures (eg., membranes and proteins), scavenging free radicals and buffering cellular redox potential under stress conditions [22]. Similar studies were carried out in other crops. Under drought stress, there was a progressive increase in free proline in cotton plants [23] and a notable proline accumulation was observed in sugar beet leaves when drought stress became severe [24]. It has been already proved that proline is having direct correlation with drought stress $[25,26]$ as indicated in the present study. Proline in general was known to correlate 
with stress tolerance and has a direct effect on tolerance capability of plant [22] supporting our present findings.

Another important biochemical parameter contributes to drought stress is nitrate reductase activity. Water deficit induces an abrupt reduction in the uptake and nitrate flux rates from roots to leaves, preventing the mechanisms of NR protein synthesis induction and NR activity. The NR activity decline during water stress is mainly attributed to low NO3 - absorption and availability resulting from water uptake deprivation [27]. The present results indicated that the tolerant hybrids had high resistance to drought stress and were able to regulate the nitrate reduction activity even with less water. Usually, drought stress reduces the enzyme activity and that is the reason the amount of reductase enzyme was low in hybrids whereas in the control, it was more. NRase is closely associated with plant growth and development [28]. It is generally accepted that drought stress has a negative impact on plant's photosynthetic activity, $\mathrm{N}$ concentrations, free amino acids or soluble protein contents accompanied with a decline of nitrate reductase activity in many plant species, such as maize [29], potato [30] and winter wheat [31]. The plants subjected to water stress produces less amount of total protein which results in a decrease in the synthesis of nitrate reductase activity caused by low nitrate flux [32]. The amount of NR enzyme generally decreases during drought stress and hence, the hybrids having more NR enzyme were more tolerant to drought stress and were able to regulate the nitrogen assimilation in plants [33]. Therefore, in the present study NR was found to be directly related to the dependent variable.

Reactive Oxygen Species (ROS) accumulation during stress greatly depends on the balance between ROS production and ROS scavenging mechanism [34]. When plants are subjected to any kind of stress, the cells have an increased production of reactive oxygen species (ROS) which in normal cases, is removed from time to time. Under stress, these become high in number and results in oxidative damage. These are removed by anti-oxidant systems which form the first line of defence which is superoxide dismutase. ROS-scavenging mechanisms were shown to have an important role in protecting plants against osmotic stresses [35].

In the experiment when plants were subjected to analysis, the tolerant and highly tolerant hybrids showed more amount of superoxide dismutase enzyme as compared to the susceptible hybrids and the control which was kept under fully irrigated condition had the least amount of SOD in it. This indicated that SOD will get accumulated under drought stress conditions and forms a defence system against the stress [36]. Superoxide dismutase
(SOD) are the enzymes that forms the first line defense and catalyses the dismutation of $\mathrm{O}^{2-}$ radicals to $\mathrm{H}_{2} \mathrm{O}$ and $\mathrm{O}_{2}$. Hence, the amount ofSOD increases with increase in stress conditions indicating it'sdirect relation to drought stress [37, 38]. Similar studies on development of water stress in Curtilobumsolanum and Solanumtuberosum due to over production of SOD in chloroplasts were also reported [39] which supported our findings.

Many plants accumulate compounds, termed compatible solutes, to cope with stress conditions. One of the most extensively studied compatible solutes is glycine betaine [40] Not only GB acts as an osmoregulator, but also stabilizes the structures and activities of enzymes and protein complexes, and maintains the integrity of membranes against the damaging effects of stress [41]. The role of glycine betaine to drought tolerance has been reported in many cases [42, 43]. Genes associated with glycine betaine synthesis in higher plants and microbes have been transferred into plants which do not accumulate glycine betaine, such as Arabidopsis thaliana [44], Brassica napus [45] Persimmon [46] and rice [47]. The metabolic engineering of glycine betaine biosynthesis in these plants improved the tolerance of transgenic plants to salt, drought and extreme temperature stresses [48]. When the levels of glycine betaine was correlated with the extent of increased tolerance, the accumulation of glycine betaine was found to be induced under stress conditions $[49,50]$.

Hence, these biochemical parameters were reliable source to detect drought stress in cocoa. However, the correlation and path analysis revealed that proline and the nitrate reductase are the two important parameter that contribute to drought tolerance. To find out the actual relationship between the dependent variables (the biochemical parameters) and the independent variable (the number of leaves retained, a binary regression analysis was carried out. The results of present study indicated that the proline and nitrate reductase can be used as biochemical indicators in cocoa drought breeding programme (Fig. 2).

\section{Conclusion}

The parameters proline and glycine betaine represented the osmolyte group whereas nitrate reductase and superoxide dismutase represented the enzyme group. In all the crosses, the content of proline was high in highly tolerant and tolerant hybrids as compared to susceptible hybrids. The control which was fully irrigated condition was having the least amount of proline. The glycine betaine also followed the same trend, as tolerant and highly tolerant hybrids were having more amount of glycine betaine as compared to susceptible 


\section{Phenes contributing to drought tolerance}

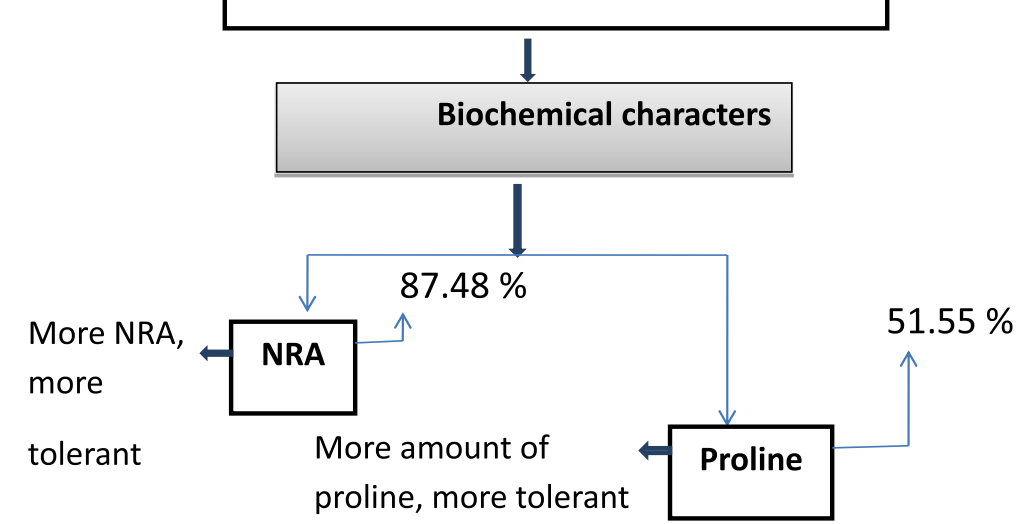

Fig. 2 Biochemical phenes and their association with drought tolerance in cocoa

hybrids and the control indicating that these two osmolytes accumulated only during water stress. In case of superoxide dismutase, the highly tolerant and tolerant hybrids were having high amount of superoxide dismutase as compared to the susceptible hybrids whereas the control was having the least amount of superoxide dismutase enzyme. In case of nitrate reductase activity, the highly tolerant and tolerant hybrids were having high amount of enzyme as compared to the susceptible hybrids. The control kept at fully irrigated condition was having the highest amount of nitrate reductase enzyme. The hybrids having high amount of nitrate reductase were more tolerant because generally, this enzyme reduces under drought stress. Based on corelation, path analysis and binary logistic regression analysis, proline and nitrate reductase can be used as reliable parameters in drought breeding programmes in the future.

\section{Abbreviations}

NR: Nitrate reductase; NRA: Nitrate reductase activity; GB: Glycine betaine; SOD: Super oxide dismutase; HT: Highly tolerant; T: Tolerant; S: Susceptible; HS: Highly susceptible; ROS: Reactive oxygen species; CRC: Cocoa Research Centre; CRD: Completely randomised design.

\section{Acknowledgments}

The author acknowledge Kerala Agricultural University and Mondelez International (Earlier Cadbury India Ltd).

\section{Authors' contributions}

Juby Baby carried out the work as a part of her post graduate programme under the guidance of Dr. Janaki Seifudeen Minimol as major advisor and the programme was undertaken under a project funded by Mondelez InternationIal. Dr. Suma Basura, Dr. Jiji Joseph and Dr. Santhoshkumar Adiyodi Venugopal as advisory committee members whose whole hearted co-operation helped to complete the project sucessfully. Dr. Panchami Pottekkat Sidharthan is the research associate associated with the project and assisted Juby Baby to complete the project. All authors read and approved the final manuscript. This manuscript is not submitted elsewhere for publication.

\section{Funding}

This study was financially supported by Mondelez International (Earlier Cadbury India Ltd) and Kerala Agricultural University, Vellanikkara.

\section{Availability of data and materials}

All data generated or analysed during this study are included in this article and its supplementary files.

\section{Declarations}

\section{Ethics approval and consent to participate}

The article does not contain any studies with human participants or animals performed by any of the authors. Plant materials used in the experiments were from Cocoa Research Centre, Vellanikkara, Thrissur. All methods in the experiment were performed in accordance with the relevant guidelines/ regulations/legislation of Kerala Agricultural University, Vellanikkara, Thrissur.

\section{Consent for publication}

Not applicable.

\section{Competing interests}

The authors declare that they have no competing interests.

\section{Author details}

${ }^{1}$ Department of Plant Breeding and Genetics, College of Agriculture, Kerala Agricultural University, Thrissur, India. ${ }^{2}$ Cocoa Research Centre, Kerala Agricultural University, Thrissur, India. ${ }^{3}$ Department of Tree Physiology and Breeding, College of Forestry, Kerala Agricultural University, Thrissur, India.

Received: 24 March 2021 Accepted: 28 September 2021

Published online: 15 December 2021

\section{References}

1. WCF. Cocoa market update. World Cocoa Foundation; 2012. Available at: https://www.worldcocoafoundation.org/wp-content/uploads/CocoaMarket-Update-as-of-.20.2012.pdf.

2. Prasannakumari AS, Vikraman Nair R, Lalithabai EK, Mallika VK, Minimol JS, Abraham K, et al. Cocoa in India. Kerala: Kerala Agricultural University; 2009. p. 72.

3. Minimol JS, Suma B, Shija TK, Shilpa KS. Genotypic and seasonal variations affecting yield of cocoa. J Agrometeorol. 2020;22:528-31.

4. Gilbert ME, Medina V. Drought adaptation mechanisms should guide experimental design. Trends Plant Sci. 2016;21:639-47. 
5. Laderach P, Martinez-Valle A, Schroth G, Castro N. Predicting the future climatic suitability for cocoa farming of the world's leading producer countries, Ghana and Cote d'Ivoire. Clim Chang. 2013;119:841-54.

6. Huang S, Van Aken O, Schwarzlander M, Belt K, Millar AH. The roles of mitochondrial reactive oxygen species in cellular signaling and stress response in plants. Plant Physiol. 2016;171:1551-9.

7. Martinez V, Nieves-Cordones M, Lopez-Delacalle M, Rodenas R, Mestre TC, Garcia Sanchez F, et al. Tolerance to stress combination in tomato plants: new insights in the protective role of melatonin. Molecules. 2018;23:535-40

8. Binimol B. Identification of drought tolerant cocoa types. Thrissur: M.SC. (Ag.) Thesis, Kerala Agricultural University; 2005. p. 95.

9. Souza CC, Oliveira FA, Silva IF, Amorim Neto MS. Evaluation of methods of available water determination and irrigation management in "Terra Roxa" under cotton crop. Rev Bras Eng Agric. 2000;4:338-42.

10. CCRP [Cadbury-KAU Co-operative Cocoa Research Project]. Annual report 2014-2015. Vellanikkara: Cocoa Research Centre; 2015. p. 153.

11. Bates $L$, Waldren RP, Teare ID. Rapid determination of free proline for water-stress studies. Plant Soil. 1973;39:205-7.

12. Evans $\mathrm{HJ}$, Nason A. Pyridine nucleotide-nitrate reductase from extracts of higher plants. Plant Physiol. 1953;28:233.

13. Dhindsa RS, Plumb DP, Thorpe TA. Leaf senescence: correlated with increased levels of membrane permeability and lipid peroxidation, and decreased levels of superoxide dismutase and catalase. J Exp Bot. 1981;32:93-101.

14. Grieve CM, Grattan SR. Rapid assay for determination of water-soluble quaternary amino compounds. Plant Soil. 1983;70:303-7.

15. Al-Jabouri RA, Miller PA, Robinson HF. Genotypic and environmental variance in upland cotton cross of interspecific origin. Agron J. 1958:50:633-7.

16. Wright S. Correlation and causation. J Agric Res. 1921;20:557-85.

17. Dewey DI, Lu KH. A correlation and path-coefficient analysis of components of crested wheatgrass seed production. Agron J. 1959;51:515-8.

18. Lenka D, Mishra B. Path coefficient analysis of yield in rice varieties. Indian J Agric Sci. 1973;43:376-9.

19. Enriquez GA. Early selection for vigour of hybrid seedlings. In: Proceedingsof the 8th international symposium on cocoa research; 1981. p. 535-9.

20. Rampino P, Pataleo S, Gerardi C, Mita G, Perrotta C. Drought stress response in wheat: physiological and molecular analysis of resistant and sensitive genotypes. Plant Cell Environ. 2006;29:2143-52.

21. Minimol JS, Amma SP. Self incompatibility assessment in vascular streak dieback (VSD) disease resistant hybrids of cocoa (Theobrama cocoa L.) Asian J Hort. 2013:8:114-7.

22. Ashraf M, Foolad MR. Roles of glycine betaine and proline in improving plant abiotic stress resistance. Environ Exp Bot. 2007;59:206-16.

23. Yi XP, Zhanga YL, Yao HS, Luo HH, Gou L, Chow WS, et al. Rapid recovery of photosynthetic rate following soil water defcit and re-watering in cotton plants (Gossypium herbaceum L.) is related to the stability of the photosystems. J Plant Physiol. 2016;193:23-34.

24. Monreal JA, Jimenez ET, Remesal E. Proline content of sugar beet storage roots: response to water defcit and nitrogen fertilization at feld conditions. Environ Exp Bot. 2007:60:257-67.

25. Mali PC, Mehta SL. Effect of drought on enzymes and free proline in rice varieties. Phytochem. 1977:16:1355-7.

26. Karamanos AJ, Drossopoulos JB, Niavis CA. Free proline accumulation during development of two wheat cultivars with water stress. J Agric Sci. 1983;100:429-39.

27. Foyer $\mathrm{CH}$, Valadier MH, Migge A, Becker TH. Drought-induced effects on nitrate reductase activity and mRNA and on the coordination of nitrogen and carbon metabolism in maize leaves. Plant Physiol. 1998;1 17:283292.

28. Ferrario-Mery S, Valadier $\mathrm{MH}$, Foyer $\mathrm{CH}$. Over-expression of nitrate reduc tase in tobacco delays drought-induced decreases in nitrate reductase activity and mRNA. Plant Physiol. 1998;117:293-302.

29. Sinha SK, Nicholas JD. Nitrate reductase in relation to drought. In: Palag LG, Aspinal LD, editors. Physiology and biochemistry of drought resistance. Sydney: Academic Press; 1981. p. 145.

30. Ghosh SC, Asanuma K, Kusutani K, Toyota M. Effects of moisture stress at different growth stages on the amount of total non-structural carbohydrate, nitrate reductase activity and yield of potato. Jpn J Trop Agr. 2000;44:158-66.
31. $X u Z Z, Y u Z W$. Nitrogen metabolism in flag leaf and grain of wheat in response to irrigation regimes. J Plant Nutr Soil Sci. 2006;169:118-26.

32. Costa RCL, Lobato AKS, Oliveira Neto CF, Maia PSP, Alves GAR, Laughinghouse HD. Biochemical and physiological responses in two Vignauguiculata(L.) walp cultivars under water stress. J Agron. 2008;7:98-101.

33. Xu ZZ, Zhou GS. Effects of water stress on photosynthesis and nitrogen metabolism in vegetative and reproductive shoots of Leymus chinensis. Photosynthetica. 2005:43:29-35.

34. Mittler R, Vanderauwera S, Gollery M, Breusegem VF. Reactive oxygen gene network of plants. Trends Plant Sci. 2004;9:490-8.

35. Abbasi AR, Hajirezaei M, Hofius D, Sonnewald U, Voll L, M. Specific roles of alpha- and gamma-tocopherol in abiotic stress responses of transgenic tobacco. Plant Physiol. 2007;143:1720-38.

36. Rizhsky L, Liang H, Shuman J, Shulaev V, Davletova S, Mittler R. When defensepathways collide, the response of Arabidopsis to a combination of drought and heat stress. Plant Physiol. 2004;134:1683-96.

37. Bowler C, Montagu MV, Inze D. Superoxide dismutase and stress tolerance. Annu Rev Plant Physiol Plant Mol Biol. 1992;43:83-116.

38. Luna CM, Pastori GM, Driscoll S, Groten K, Bernard S, Foyer CH. Drought controls on $\mathrm{H}_{2} \mathrm{O}_{2}$ accumulation, catalase (CAT) activity and CAT gene expression in wheat. J Exp Bot. 2004;56:417-23.

39. Martinez CA, Loureiro ME, Oliva MA, Maestri M. Differential responses of superoxide dismutase in freezing resistant Solanumcurtilobum and freezing Solanumsensitive tuberosum subjected to oxidative and water stress. Plant Sci. 2001;160:505-15.

40. Rathinasabapathi B. Metabolic engineering for stress tolerance: installing osmoprotectants synthesis pathways. Ann Bot. 2000;86:709-16.

41. Sakamoto A, Murata N. The role of glycine betaine in the protection of plants from stress: clues from transgenic plants. Plant Cell Environ. 2002;25:163-71.

42. Robinson SP, Jones GP. Accumulation of glycine betaine in chloroplasts provides osmotic adjustment during salt stress. Funct Plant Biol. 1986;13:659-68.

43. Genard H, Saos J, Billard JP, Tremolieres A, Boucaud J. Effect of salinity on lipid composition, glycine betaine content and photosynthetic activity in chloroplasts of Suaeda maritima. Plant Physiol Biochem. 1991;29:421-7.

44. Alia H, Sakamoto A, Murata N. Enhancement of the tolerance of Arabidopsis to high temperatures by genetic engineering of the synthesis of glycine betaine. Plant J. 1998;16:155-61.

45. Huang J, Hirji R, Adam L, Rozwadowski KL, Hammerlind JK, Keller WA, et al. Genetic engineering of glycine betaine production towards enhancing stress tolerance in plants: metabolic limitations. Plant Physiol. 2000;122:747-56.

46. Gao M, Sakamoto A, Miura K, Murata N, Sugiura A, Tao R. Transformation of Japanese persimmon (Diospyros kaki Thunb.) with a bacterial gene for choline oxidase. Mol Breed. 2000;6:501-10.

47. Mohanty A, Kathuria H, Ferjani A, Sakamoto A, Mohanty P, Murata N, et al. Transgenics of an elite indica rice variety Pusa Basmati 1 harbouring the coda gene are highly tolerant to salt stress. Theor Appl Gen. 2002;106:51-7.

48. Sulpice R, Tsukaya H, Nonaka H, Mustardy L, Chen TH, Murata N. Enhanced formation of flowers in salt-stressed Arabidopsis after genetic engineering of the synthesis of glycine betaine. Plant J. 2003;36:165-76.

49. Yang WJ, Rich PJ, Axtell JD, Wood KV, Bonham CC, Ejeta G, et al. Genotypic variation for glycine betaine in sorghum. Crop Sci. 2003;43:162-9.

50. Park EJ, Jeknic Z, Sakamoto A, DeNoma J, Yuwansiri R, Murata N, et al. Genetic engineering of glycine betaine synthesis in tomato protects seeds, plants, and flowers from chilling damage. Plant J. 2004;40:474-87.

\section{Publisher's Note}

Springer Nature remains neutral with regard to jurisdictional claims in published maps and institutional affiliations. 